\title{
AUTOMATIC SEGMENTATION OF PELVIS FOR BRACHYTHERAPYOF PROSTATE
}

Martin Kardell, Maria Magnusson, Michael Sandborg, Gudrun Alm Carlsson, Julius Jeuthe and Alexandr Malusek

\section{Linköping University Post Print}

\section{Tweet}

N.B.: When citing this work, cite the original article.

Original Publication:

Martin Kardell, Maria Magnusson, Michael Sandborg, Gudrun Alm Carlsson, Julius Jeuthe and Alexandr Malusek, AUTOMATIC SEGMENTATION OF PELVIS FOR BRACHYTHERAPYOF PROSTATE, 2015, Radiation Protection Dosimetry. http://dx.doi.org/10.1093/rpd/ncv461

Copyright: Oxford University Press (OUP): Policy B - Oxford Open Option A http://www.oxfordjournals.org/

Postprint available at: Linköping University Electronic Press http://urn.kb.se/resolve?urn=urn:nbn:se:liu:diva-122978 


\section{Automatic segmentation of pelvis for brachytherapy of}

\section{prostate}

M. Kardell ${ }^{1}$, M. Magnusson ${ }^{1,2}$, M. Sandborg ${ }^{1}$, G. Alm Carlsson ${ }^{1}$, J. Jeuthe ${ }^{1}$ and A. Malusek ${ }^{1}$

${ }^{1}$ Medical Radiation Physics, Department of Medical and Health Sciences and Center for

Medical Image Science and Visualisation, Linköping University, SE-58185 Linköping,

Sweden

${ }^{2}$ Computer Vision Laboratory, Department of Electrical Engineering, Linköping University, SE-58183 Linköping, Sweden

Corresponding author: alexandr.malusek@liu.se

fax +46101032895

phone +46101033059

Short running title (max 40 characters): Automatic segmentation of pelvis 
Automatic segmentation of pelvis for brachytherapy of prostate

M. Kardell, M. Magnusson, M. Sandborg, G. Alm Carlsson, J. Jeuthe and A. Malusek

\begin{abstract}
Advanced model-based iterative reconstruction algorithms in quantitative computed tomography perform automatic segmentation of tissues to estimate material properties of the imaged object. Compared to conventional methods, these algorithms may improve quality of reconstructed images and accuracy of radiation treatment planning. Automatic segmentation of tissues is, however, a difficult task. The aim of this work is to develop and evaluate an algorithm that automatically segments tissues in CT images of the male pelvis. The newly developed algorithm (MK2014) combines histogram matching, thresholding, region growing, deformable model and atlas based registration techniques for the segmentation of bones, adipose tissue, prostate and muscles in CT images. Visual inspection of segmented images showed that the algorithm performed well for the five analysed images. The tissues were identified and outlined with accuracy sufficient for the dual-energy iterative reconstruction algorithm (DIRA) whose aim is to improve the accuracy of radiation treatment planning in brachytherapy of the prostate.
\end{abstract}




\section{INTRODUCTION}

Model-based iterative reconstruction (MBIR) algorithms $^{(1)}$ in quantitative computed tomography $(\mathrm{CT})$ have the potential to improve the accuracy of radiation treatment planning by removing artefacts (for instance beam hardening and scatter) from reconstructed images. Physical realism of their models determines the accuracy of measured data corrections that are calculated by simulating the imaging system. Ideally, advanced MBIR algorithms will automatically classify all body tissues. This task is, however, very difficult. One way to reach that goal is an automatic segmentation of reconstructed images.

Segmentation requires anatomical knowledge and thus is typically done by a trained medical professional, e.g. a radiologist. The process is labour intensive and the results often differ depending on the expertise and personal preferences of the person ${ }^{(2,3)}$. Current automatic segmentation algorithms may fully automate the process, but they rarely provide results directly usable in clinical practice. Instead, semi-automated algorithms where an operator sets initial conditions and boundaries or gives feedback between iterations are often used.

Segmentation of tissues in the male pelvis is difficult because of (i) large variability in the shape and size of the bones with slice position and (ii) small difference between Hounsfield values of pelvic soft tissues. The most common approach is to segment one tissue at the time, usually only the prostate and sometimes the rectum or bladder. Recent segmentation methods use atlases or masks that are registered or deformed to match the image to be segmented, for instance the rigid registration in $3 \mathrm{D}$ on $\mathrm{CT}$ images ${ }^{(4)}$, multi atlas non-rigid registration, active appearance model, probabilistic active shape model ${ }^{(5)}$, and deformable models ${ }^{(6,7)}$.

Direct application of the approaches described above is limited for advanced MBIR algorithms as they require automatic segmentation of all tissues in the imaged slice. The authors developed 
an MBIR dual-energy iterative reconstruction algorithm (DIRA) ${ }^{(8)}$ whose primary application is in brachytherapy. The question is whether it is possible to combine already existing segmentation techniques to an automatic segmentation algorithm that can be used in DIRA for the brachytherapy of prostate. The aim of this work is to develop such an algorithm.

\section{MATERIALS AND METHODS}

An automatic algorithm for the segmentation of CT images of male pelvis (MK2014) was developed. Its description and evaluation follow.

\section{Segmentation algorithms}

The MK2014 algorithm uses histogram matching, thresholding, region growing, deformable model and atlas based registration techniques. Prior to the application of these methods, three transformations of image intensities were performed: (i) CT numbers lower than -524 HU were set to $-1000 \mathrm{HU}$ to replace with air the blankets covering the patient. (ii) $\mathrm{CT}$ numbers were rescaled to the intensity range from 0 to 255 ; the minimum and maximum $\mathrm{CT}$ numbers in the image corresponded to the intensities of 0 and 255, respectively. (iii) The intensity of 0 was set to -100 to increase the contrast between subcutaneous fat and surrounding air.

Histogram matching ${ }^{(9)}$ adjusts intensities of the input image so that the cumulative distribution functions of the input and reference image intensities approximately match. The method suppresses variability in image intensities caused by different tube voltages and, in case of a properly selected reference image, improves performance of region growing by enhancing contrast between adjacent tissues. The MATLAB's function imhistmatch was used.

Thresholding $^{(9)}$ selects pixels in a certain intensity range; pixels with other intensities are removed. The result is a binary image. In this work, regular thresholding with fixed ranges implemented by the MATLAB's function histc was used. Ranges for air, adipose tissue and 
compact bone were set to $[0,6],[6,75]$ and $[180,255]$, respectively, in the intensity scale from 0 to 255 ; these values provided best results for the five analysed images.

Region growing ${ }^{(10)}$ is an intensity based segmentation method that enlarges a region as follows. The region starts with one or several seeds, each consisting of one or several pixels. The seeds are manually or automatically positioned inside the tissue of interest (TOI). The intensity of the seed is compared to the intensity of neighbouring pixels and if the intensity of the latter is inside a predefined acceptance range, the neighbouring pixels are added to the growing region. The growing and comparison continues with the only change that the neighbouring pixel intensity is compared to the mean intensity of the growing region. When no pixels neighbouring the growing region have an intensity close enough to the mean intensity of the region, the growing stops and the algorithm is completed. A multi-pixel multi-seed method based on Franiatté's simple single-seeded growing algorithm ${ }^{(11)}$ was implemented. The method was used for the segmentation of bones and adipose tissue. Seeds were automatically generated using the thresholding combined with an image erosion (MATLAB's bwulterode) and removal of noise artefacts (MATLAB's bwmorph). In case of adipose tissue, the image was divided to $4 \mathrm{x} 4$ equally sized segments and the seeds were searched for in each segment.

Atlas based segmentation is a method where a reference image whose TOIs are already segmented (an atlas) is transformed to represent the TOIs in the processed CT image. In MK2014, several atlases are provided to cover the variability in bone shapes and positions in the pelvic region. In some cases, the user may need to create additional atlases. The initial position of the atlas was set so that the outline contour of the atlas body matched the outline contour of the target patient's body; this transformation is called the initial translation and scaling in figure 1 . No other transformation was used for the segmentation of muscles. For the segmentation of prostate, an additional affine transformation was used; it was defined so that bones from the atlas were registered to the already segmented bones, other tissues were 
ignored. The registration was implemented according to Granlund and Knutsson ${ }^{(12)}$ and Svensson et $a l^{(13)}$.

The deformable model was based on the active contours without edges method ${ }^{(14)}$, which is implemented in the MATLAB's function activecontour. It uses a contour that can both grow and shrink according to an energy minimizing formula. A level set part of the algorithm stops the contour at the boundaries of the TOI. It makes the method less dependent on the image gradient. The deformable model was used for the segmentation of muscles (in this case gluteus maximus) and prostate, whose initial contours were defined by the atlas based segmentation.

\section{The MK2014 segmentation algorithm}

The MK2014 algorithm automatically segments CT images of a male pelvis to bone, adipose tissue, prostate, muscles and remaining soft tissue (figure 1) using the following steps:

1) Histogram matching adjusts image intensity.

2) Thresholding selects likely positions of bones and adipose tissue.

3) Seed generation selects positions of seed points for region growing in bones and adipose tissue using results from step 2.

4) Region growing segments bones and adipose tissue using seeds from step 3.

5) Region growing and thresholding results are combined with morphological skeleton and morphological closing.

6) Initial translation and scaling registers an atlas to the image.

7) Affine registration is performed for the prostate. It is defined so that it transforms the bones of the atlas to the bones of the image.

8) Deformable model segments the prostate and gluteus maximus muscles.

The algorithm has been implemented as an extension in the DIRA code, which is available on GitHub $^{(15)}$. 


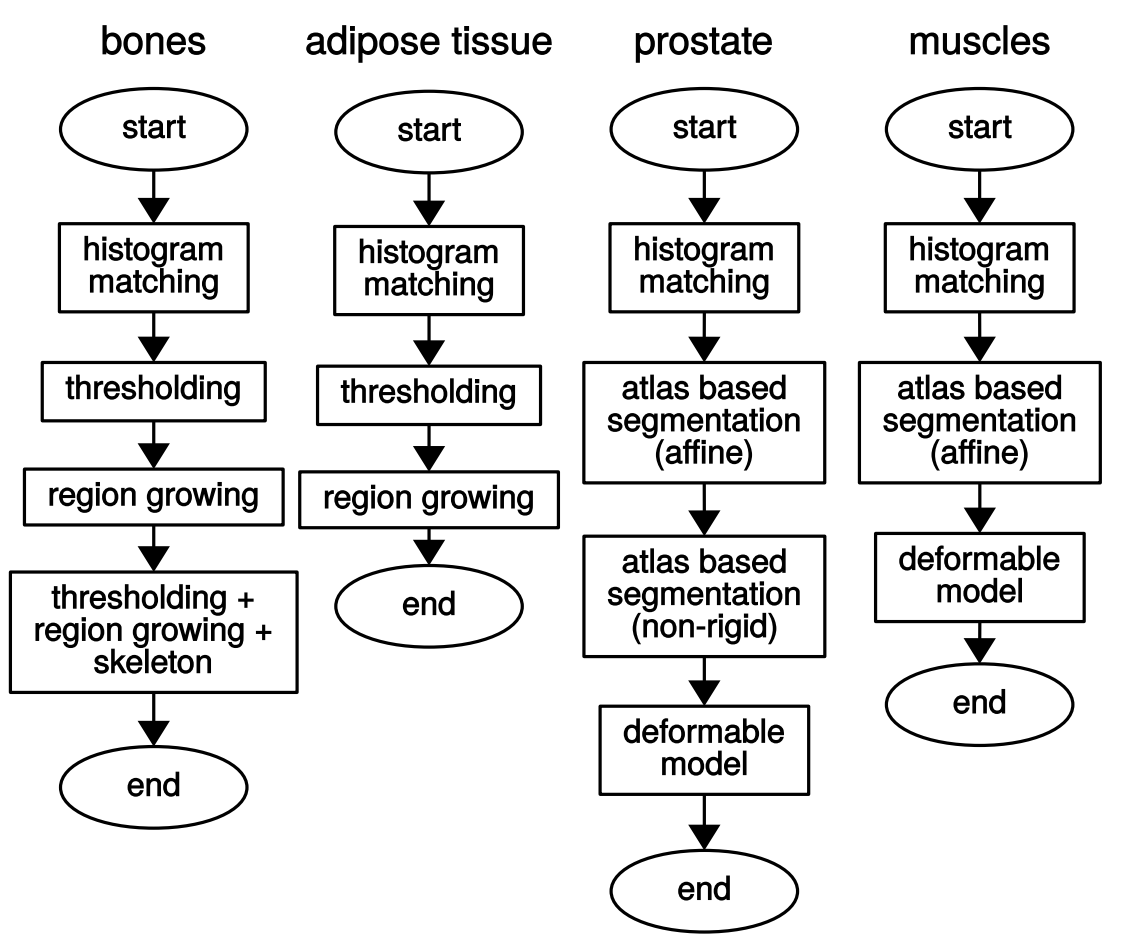

Figure 1. The segmentation of bones, adipose tissue, muscle and prostate in the MK2014 algorithm.

\section{Evaluation of the MK2014 algorithm}

Five CT images of the male pelvis at the approximate height of the hip joint were analysed. Images 1 and 2 in figure 6 were obtained from the same subject to account for the variability of the images on slice position. Images 3-5 were obtained from different subjects. Slice thicknesses were $5.0 \mathrm{~mm}$ and $2.5 \mathrm{~mm}$ for images 1-3 and 4-5, respectively. Scans were taken at the tube voltage of $120 \mathrm{kV}$, and reconstructed images had the size of $512 \times 512$ pixels.

The quality of segmentation of the five images was checked using visual assessment. Moreover for image 3 the Dice similarity coefficient ${ }^{(16)}$ (DCS) was used. The DSC, D, for a segmented tissue was defined as

$$
D=\frac{2|A \cap B|}{|A|+|B|},
$$


where $A$ and $B$ are sets of pixels classified as the tissue from the automatic segmentation and ground truth, respectively, and $|\cdot|$ denotes the number of pixels in the set. Values of $D$ are between 0 (no overlap) and 1 (perfect overlap). The ground truth for the image was determined by an experienced radiologist.

Performance of the MK2014 algorithm was evaluated in MATLAB R2014a on an Intel Core i5-4200U CPU with 8 GiB RAM.

\section{RESULTS}

For bones, both thresholding and region growing were used. To eliminate errors caused by statistical noise and artefacts in the image, the former method was complemented with a removal of small objects (MATLAB's bwareaopen) and both methods were complemented with filling of small holes (MATLAB's imfill and bwareaopen). Such enhanced thresholding performed better for bones in the upper part of the image, while the enhanced region growing performed better for the tailbone, see figures 2(a) and 2(b). Results from these two methods were combined by using a union of the pixels, see figure 2(c). Existing holes were closed as follows: morphological skeleton was obtained by eroding the already segmented bone (MATLAB's bwmorph). The resulting medial axis was morphologically closed (MATLAB's imclose and imfill) and united with pixels in figure 2(c), see figure 2(d). The result well matched the ground truth of the tested image, the DSC was 0.94 . 

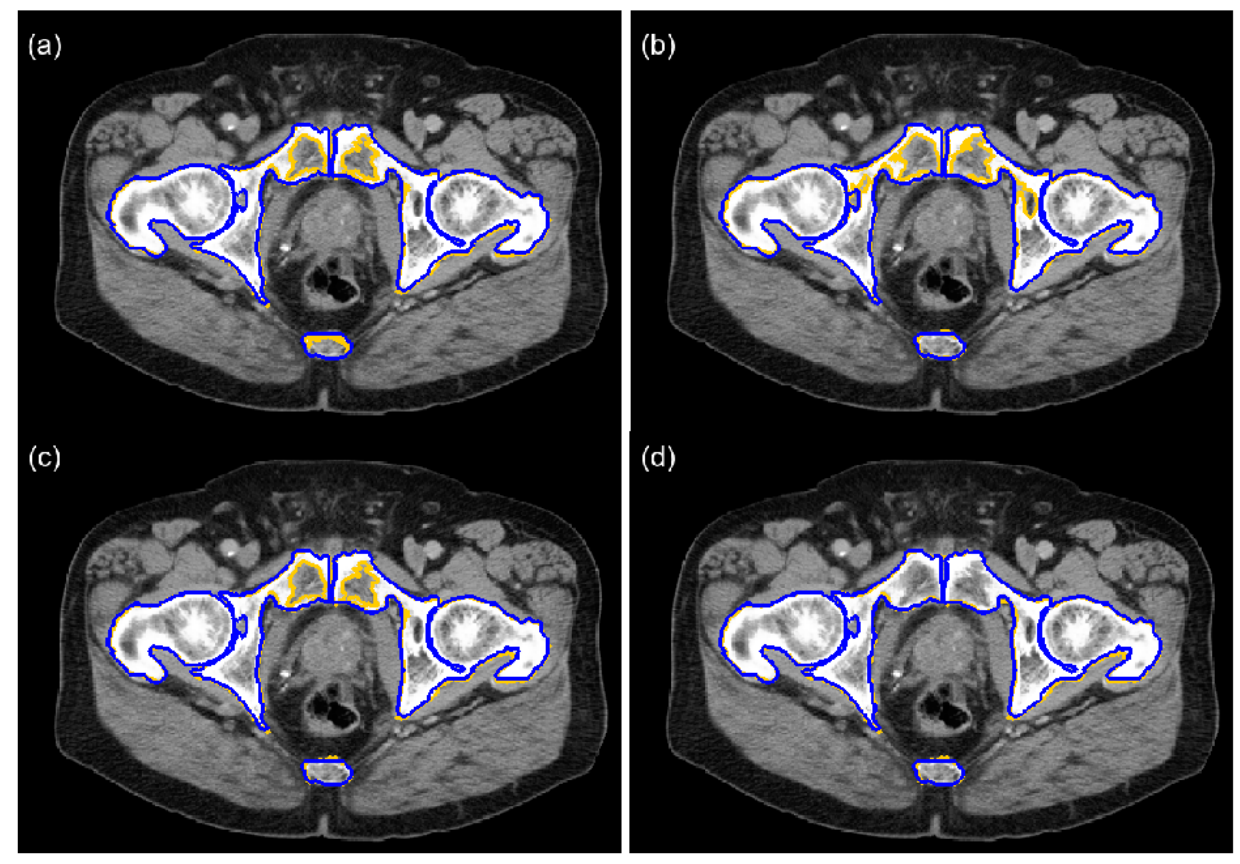

Figure 2. Automatic segmentation of bones (yellow curves) and corresponding ground truth (blue curves). (a) Thresholding with small object removal and small hole filling. (b) Region growing with small hole filling. (c) Union of the enhanced thresholding and region growing methods. (d) The result after the additional application of morphological skeleton and morphological closing.

Adipose tissue in the pelvic region consists mainly of subcutaneous fat forming a ring around the body and visceral fat inside the pelvic cavity. Figure 3 shows the subcutaneous fat segmented via the region growing and manual segmentation methods. The latter was used as the ground truth; the DSC was 0.92 . It should be noted that the ground truth was affected by uncertainties in the upper part of the image as the manual segmentation in this region strongly depended on the radiologist's preferences. 


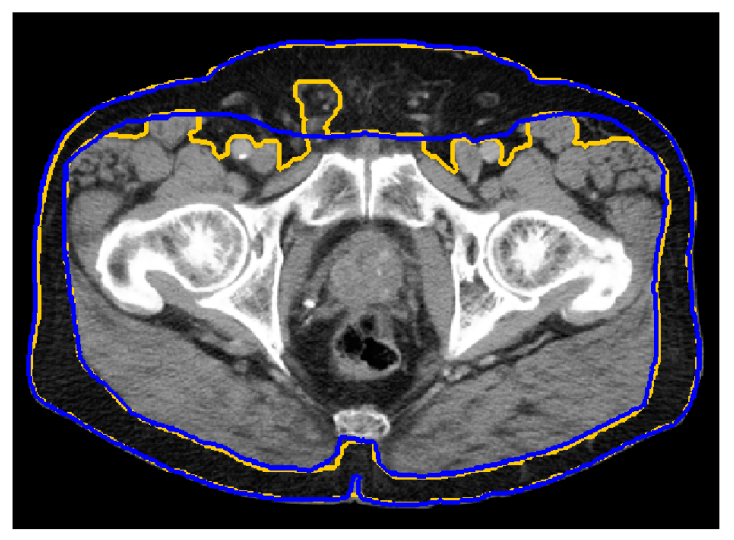

Figure 3. Automatic segmentation of adipose tissue (yellow curve) and corresponding ground truth (blue curve).

The segmentation of muscles (gluteus maximus) was complicated by their texture-like structure caused by small regions of fat inside the muscle tissue. Many segmentation methods failed in this case. Only the atlas based segmentation combined with the deformable model gave sufficiently good results, see figure 4 . The current version of the deformable model worked better when the initial state of the active contour was derived from an atlas muscle that was smaller than the target muscle. This way, the active contour grew to the target contour. Otherwise the active contour could converge to other well defined edges in the image, for instance the edges of bones.

Figure 5 shows the segmentation of image 5 using three different atlases. An inappropriate atlas lead to suboptimal segmentation of the muscle, for instance the large muscles in the atlas image 5(a) lead to oversized segmented muscles in the image 5(d); the deformable model did not reduce the size of the region. The position of the prostate in image 5 was slightly above the position predicted by the atlases. This discrepancy lead to oversized prostate region in images 5(d) and 5(f). 

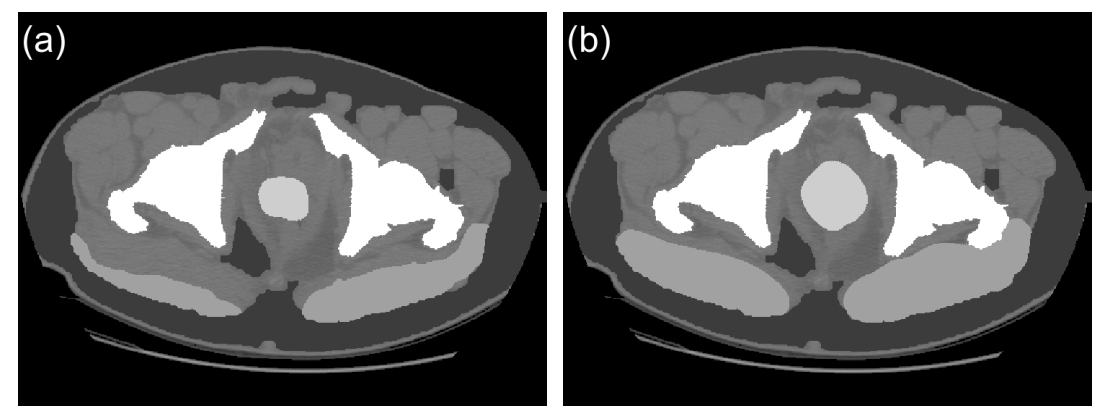

Figure 4. Segmentation of gluteus maximus muscles. (a) Atlas based segmentation using initial translation and scaling resulted in regions that were smaller than the target muscles. (b) The additional deformable model segmentation grew the region to a more correct size. Segmented tissues: bones, prostate, muscles and adipose tissue (in white to black grey level order).
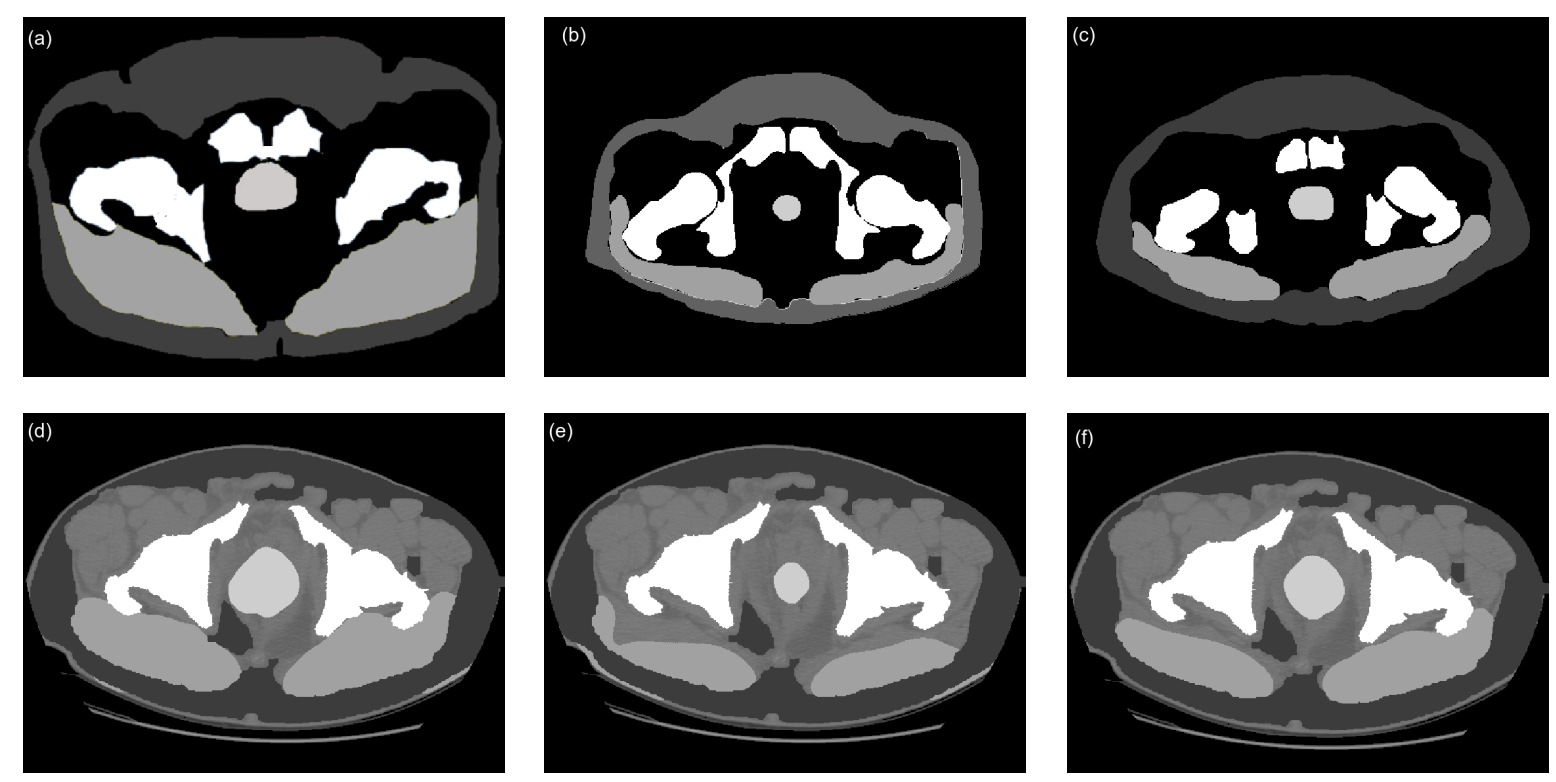

Figure 5. Atlases (top row) and corresponding segmented tissues of image 5 (bottom row).

Segmented tissues of the five analysed images are in figure 6. DSC of the four segmented TOIs in image 3, for which the ground truth was available, are in table 1. As already mentioned, the ground truth segmentation depended on the radiologist's preferences and so these numbers are associated with uncertainties that are difficult to estimate. The segmentation took about 45 seconds on the Intel Core i5-4200U CPU. 
Table 1. Quality of the automatic segmentation of image 3 estimated via the Dice similarity coefficient for bones, adipose tissue, muscles and prostate.

\begin{tabular}{llll}
\hline bones & adipose tissue & muscles & prostate \\
\hline 0.94 & 0.92 & 0.88 & 0.85 \\
\hline
\end{tabular}

1
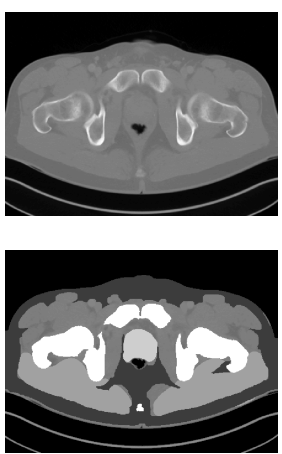

2
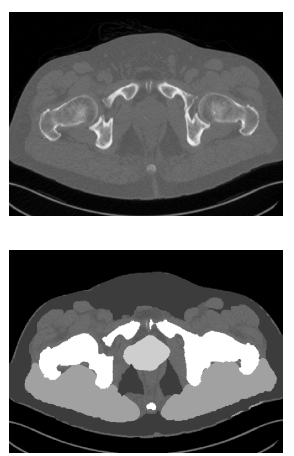

3
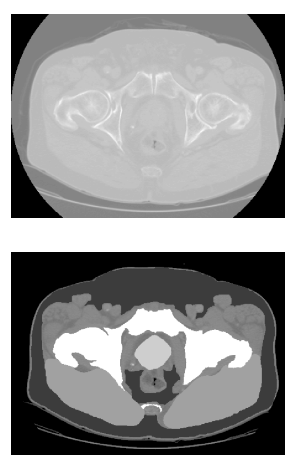

4
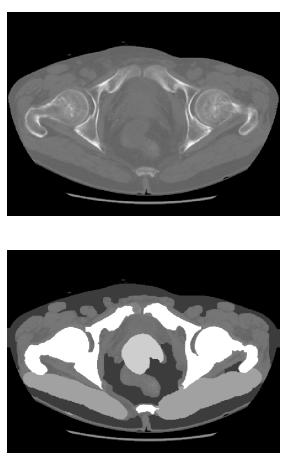

5
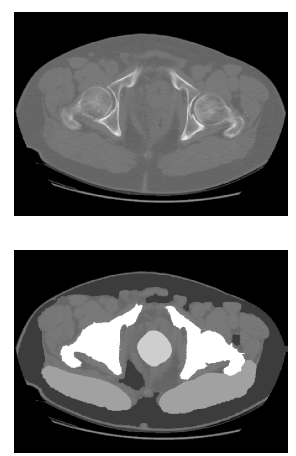

Figure 6. Original images (top row) and corresponding segmented tissues (bottom row).

Atlases 1 and 3 (figure 5) were used for images 1-3 and 4-5, respectively.

\section{DISCUSSION}

Methods originally considered for the MK2014 algorithm were: thresholding (including fuzzy C-means thresholding), region growing, watershed, graph cuts $^{(17)}$, grab cut ${ }^{(18)}$, distance regularized level set evaluation ${ }^{(19)}$, a simplified version of active contours ${ }^{(20)}$, and atlas based image segmentation. None of the considered methods performed sufficiently well alone on the test images. Some methods were not well suited for the task and had to be excluded. The remaining methods had to be combined. It should be noted that it was not possible to evaluate the methods for all values of their parameters and so some of the excluded methods may perform well for a particular task. However it is very unlikely that any of those methods would be able to accurately segment all tissues in the CT images.

The region growing algorithm was sensitive to the setting of the intensity acceptance range. The value had to be large enough so that pixels affected by image noise were included in the 
region, but it had to be lower than the contrasts between the region and adjacent tissues. The default values in the MK2014 algorithm worked well for all analysed images. In some cases, however, the user may need to use a different reference image in histogram matching to adjust the contrasts. For adipose tissue, the region growing algorithm produced similar results to the thresholding combined with hole filling. The MK2014 algorithm implemented the former, but the authors have no strong preference for either of the two methods.

The presented method worked well when the evaluated images were similar to the atlas image. As the shape and position of bones in the pelvic region vary rapidly with the z-position of the slice and the tilt of the gantry, a good match between the evaluated image and the atlas may be difficult to achieve. Problems may also arise when the prostate is notably shifted in the pelvic cavity owing to, for instance, a full urinary bladder. When segmentation using the default atlas fails, the user can chose a more suitable atlas image from a predefined set of images. A fully automatic image registration is difficult in 2D. A 3D registration algorithm may be needed if similar positioning of the patient cannot be achieved.

Simulations by Malusek et $a l^{(21)}$ showed that DIRA is not very sensitive to the selection of the base material triplet characterising pelvic soft tissues. For instance the (lipid, protein, water) and (adipose tissue, muscle, water) triplets performed similarly for soft tissues. Hence it is expected that DIRA will not be very sensitive to reasonably small inaccuracies in the segmentation of normal adipose tissue, muscle, or prostate. For instance a triplet for adipose tissue may work well for the muscle too. On the other hand, the segmentation of prostate in the MK2014 algorithm will allow the usage of base materials that better describe calcifications, for instance the (prostate tissue, calcium) doublet. This may notably increase the accuracy of DIRA. Quantitative evaluation of the benefit of the MK2014 algorithm for these cases is planned. 


\section{CONCLUSIONS}

Visual inspection of segmented images showed that the MK2014 algorithm performed well for the five analysed images. Bones, adipose tissue, muscles and prostate were identified and outlined with accuracy sufficient for DIRA. A drawback of the algorithm is its long computation time compared to the simple but less accurate thresholding-based segmentation algorithm originally used in DIRA.

\section{FUNDING}

This work was supported by the Swedish Cancer Foundation [grant numbers CAN 2012/764 and CAN 2014/691]; Medical Faculty, Linköping University; and ALF Grants, Region Östergötland [grant number LiO-438731].

\section{REFERENCES}

1. Beister, M., Kolditz, D. and Kalender, W.A. Iterative reconstruction methods in X-ray CT. Physica Medica 28, 94-108 (2012).

2. Smith, W.L., Lewis, C., Bauman, G., Rodrigues, G., D’Souza, D., Ash, R., Ho, D., Venkatesan, V., Downey, D. and Fenster, A. Prostate volume contouring: A 3D analysis of segmentation using $3 D T R U S, C T$, and MR. International Journal of Radiation Oncology Biology Physics 67, 1238-1247 (2007).

3. Rasch, C., Barillot, I., Remeijer, P., Touw, A., van Herk, M. and Lebesque, J.V. Definition of the prostate in CT and MRI: a multi-observer study. International Journal of Radiation Oncology Biology Physics 43, 57-66 (1999).

4. Boydev, C., Pasquier, D., Derraz, F., Peyrodie, L., Taleb-Ahmed, A. and Thiran, J.-P. Automatic prostate segmentation in cone-beam computed tomography images using 
rigid registration. In: 35th Annual International Conference of the IEEE Engineering in Medicine and Biology Society (EMBC). 3993-3997 (2013).

5. Litjens, G., Toth, R., van de Ven, W., Hoeks, C., Kerkstra, S., van Ginneken, B., Vincent, G., Guillard, G., Birbeck, N., Zhang, J., Strand, R., Malmberg, F., Ou, Y., Davatzikos, C., Kirschner, M., Jung, F., Yuan, J., Qiu, W., Gao, Q., Edwards, P. “Eddie," Maan, B., van der Heijden, F., Ghose, S., Mitra, J., Dowling, J., Barratt, D., Huisman, H. and Madabhushi, A. Evaluation of prostate segmentation algorithms for MRI: The PROMISE12 challenge. Medical Image Analysis 18, 359-373 (2014).

6. Chen, S., Lovelock, D.M. and Radke, R.J. Segmenting the prostate and rectum in CT imagery using anatomical constraints. Medical Image Analysis 15, 1-11 (2011).

7. Liu, X., Langer, D.L., Haider, M.A., Van der Kwast, T.H., Evans, A.J., Wernick, M.N. and Yetik, I.S. Unsupervised segmentation of the prostate using MR images based on level set with a shape prior. In: Annual International Conference of the IEEE Engineering in Medicine and Biology Society 2009, 3613-3616 (2009).

8. Magnusson, M., Malusek, A., Muhammad, A. and Alm Carlsson, G. Iterative reconstruction for quantitative tissue decomposition in dual-energy CT. In: Heyden, A., Kahl, F. (Eds.), Image Analysis. Springer Berlin Heidelberg, Berlin, Heidelberg. 479-488 (2011).

9. Gonzalez, R.C. and Woods, R.E. Digital image processing. Prentice Hall, Upper Saddle River, N.J. (2002).

10. Rogowska, J. Overview and fundamentals of medical image segmentation. In: BANKMAN, I.N. (Ed.), Handbook of Medical Imaging, Biomedical Engineering. Academic Press, San Diego, 69-85 (2000). 
11. Franiatte, S. Matlab Cenral: Simple single-seeded region growing. Available at: http://www.mathworks.com/matlabcentral/fileexchange/35269-simple-single-seededregion-growing (2015-01-16, date last accessed)

12. Granlund, G.H. and Knutsson, H. Signal processing for computer vision. Kluwer Academic Publishers, Dordrecht; Boston (1995).

13. Svensson, B., Pettersson, J., Eklund, A. and Knutsson, H. Image registration. Available at: https://www.imt.liu.se/edu/courses/TBMI02/pdfs/registration.pdf (7 October 2014, date last accessed).

14. Chan, T.F. and Vese, L.A. Active contours without edges. Image processing, IEEE transactions on 10, 266-277 (2001).

15. Malusek, A. GitHub: cmiv-dira. Available at: https://github.com/AlexandrMalusek/cmiv-dira/ (30 September 2015, date last accessed).

16. Dice, L.R. Measures of the amount of ecologic association between species. Ecology 26, $297(1945)$.

17. Boykov, Y.Y. and Jolly, M.-P. Interactive graph cuts for optimal boundary \& region segmentation of objects in N-D images. In: Eighth IEEE International Conference on Computer Vision, 2001. vol. 1, 105-112 (2001).

18. Vezhnevets, V. and Konouchine, V. GrowCut: Interactive multi-label ND image segmentation by cellular automata. In: Proc. of Graphicon. 150-156 (2005).

19. Li, C., Xu, C., Gui, C. and Fox, M.D. Distance regularized level set evolution and its application to image segmentation. IEEE Transactions on Image Processing 19, $3243-3254$ (2010). 
20. Kardell, M. Automatic segmentation of tissues in CT images of the pelvic region. Master's thesis, Linköping University (2014). Available at: http://urn.kb.se/resolve?urn=urn:nbn:se:liu:diva-112540

21. Malusek, A., Magnusson, M., Sandborg, M., Westin, R. and Alm Carlsson, G. Prostate tissue decomposition via DECT using the model based iterative image reconstruction algorithm DIRA. In: Medical Imaging 2014: Physics of medical imaging. Eds. Bruce R. Whiting; Christoph Hoeschen; Despina Kontos, SPIE International Society for Optical Engineering, Vol. 9033, 90333H-1-90333H-9 (2014). 